Ann. Zootech., I979, 28 (3), 3I5-323.

\title{
Influence du verrat sur les principaux paramètres de la productivité du troupeau et sur la durée de gestation
}

\author{
G. UZU \\ avec la collaboration technique de M. BONNEAU et J. LEBOST \\ Station de Recherches sur l'Élevage des Porcs \\ [Centre national de Recherches zootechniques, I.N.R.A. \\ $7835^{\circ}$ Jouy-en-Josas (France)
}

\begin{abstract}
Résumé
L'effet exercé par le verrat sur les principaux paramètres de la productivité d'un troupeau de race Large White a été estimé sur un ensemble de I 74I saillies et I I 34 portées. Ces données sont issues de 15 verrats utilisés en saillie naturelle dans le troupeau expérimental de La Minière au cours de la période 1972-1978.

L'influence du verrat est hautement significative sur le taux de fertilité, significative sur la taille de la portée, à la naissance et au sevrage (fig. I). Les différences de fertilité se traduisent par une variabilité de 1,6 porcelet sevré par truie et par an, celles de prolificité par 3,5 porcelets, et la combinaison des écarts de fertilité et de prolificité entre les verrats extrêmes est de 5,5 porcelets.

L'effet paternel est également hautement significatif sur la durée de gestation des truies avec une étendue de $1,30 \mathrm{j}$.
\end{abstract}

\section{Introduction}

L'examen des statistiques relatives au cheptel des reproducteurs porcins en France montre qu'il existe I verrat pour I 8 truies (Eurostat, I978), engendrant en moyenne 360 descendants par an. L'impact économique d'un mâle stérile, ou même subfertile, peut donc être considérable.

La variabilité des performances de reproduction entre les verrats a été clairement démontrée en insémination artificielle et son étendue est estimée à 2,5 porcelets par portée dans 1'étude de LEGAuLiT et OLIIVIER (I965). Par contre, en saillie naturelle, pratiquée dans plus de $95 \mathrm{p}$. Ioo des élevages français, le nombre de portées par verrat est limité et souvent insuffisant pour détecter des différences entre les reproducteurs. Ainsi, l'effet exercé par le mâle sur la productivité numérique d'un troupeau est-il encore controversé. Le verrat n'aurait aucune influence 
sur la taille de la portée selon de nombreux auteurs : KRALLINGER et SchotT (I934), Dzaparidze (I935), Musson (I946), REDdy, Lasley et Mayer (I958), Wirson et al. (I962), LEgault (I970), Rico et Menchaca (I975), Smith et Tof'T (1978). Au contraire, d'autres études, moins nombreuses, démontrent l'influence significative du verrat sur la taille de la portée en monte naturelle : BARBosA (I962), SKJERVOLD (I963).

L'objectif de cette étude est d'estimer avec précision 1'effet direct des verrats utilisés en saillie naturelle sur la fertilité et la prolificité des truies et d'en mesurer les conséquences sur la productivité du troupeau, produit des deux paramètres précédents. L'influence du verrat sur le poids moyen des porcelets à la naissance et sur la durée de gestation des truies est également pris en compte.

\section{Matériel et méthodes}

L,es données sont collectées sur le troupeau de race Large White du domaine expérimental de la Minière et comprennent un ensemble de I 74I saillies simples et I I34 portées. Nous avons comparé les performances de I5 verrats Large White ayant effectué chacun entre 55 et 223 saillies au cours de la période I972-I978. Le rythme des saillies est de I à 2 par semaine et ne diffère pas entre les reproducteurs.

Six variables ont été considérées dans les analyses :

- le taux de fertilité estimé par le pourcentage de non-retour, $2 \mathrm{I}$ jours après la saillie;

- la taille de la portée calculée à la naissance (nés totaux et nés vivants) et au sevrage (entre $2 \mathrm{I}$ et $30 \mathrm{j}$ );

- le poids moyen des porcelets à la naissance;

- la durée de gestation.

L'influence du verrat sur le taux de fertilité a été analysée par un test de comparaison des fréquences (test $2 \widehat{\mathrm{I}}, \mathrm{A}$ RBONNIER, I966). Les 5 autres variables ont été classées par verrat, par période de mise-bas ayant chacune une durée de 2 mois, et par numéro de portée (5 classes de I à 5 et plus). Les effets attachés à chacun des niveaux des 3 facteurs ont été estimés par la méthode des moindres carrés, après avoir réalisé le test de 1'hypothèse de non-interaction. Les calculs ont été faits sur ordinateur selon la méthode de STEVENS (I95I). Les variables ont été représentées par le mođèle mathématique suivant : yijkl $=u+v i+$ $a j+o k+s i j k l$, avec :

- yijkl : variable étudiée;

- $u$ : moyenne générale;

- vi : effet du $i^{\text {ème } ~ " v e r r a t ~} "$ avec $i=$ I à I5;

- $a j \quad$ : effet de la $j$ ème "période de mise-bas " avec $j=\mathrm{I}$ à 6 ;

- ok : effet du $k^{\text {ème }}$ " numéro d'ordre de la portée " avec $k=\mathrm{I}$ à 5 ;

- sijkl : erreur aléatoire de moyenne nulle et de variance $\sigma^{2}$.

L'analyse de la durée de gestation a été reprise selon un même modèle, mais avec les facteurs de variation suivants : effets "verrats ", "numéro de portée " et " taille de la portée " à la naissance (5 classes). 


\section{Résultats}

Le tableau I présente les valeurs moyennes des principales mesures effectuées.

\section{a. - Infuence du verrat sur le taux de fertilité (fig. I)}

L'influence du verrat sur la fertilité est hautement significative. Ainsi, un verrat peut être considéré arbitrairement comme subfertile avec un taux de fertilité de $5^{8}$ p. I0o, et il est possible de classer les autres animaux en 2 groupes, ayant respectivement des taux de l'ordre de 73 à 86 p. гоo.

TABLEAU I

Valeurs moyennes des paramètres de reproduction du troupeau Reproduction parameters observed in the pig herd

\begin{tabular}{|c|c|c|c|}
\hline $\begin{array}{l}\text { Variables } \\
\text { Variates }\end{array}$ & $\begin{array}{l}\text { Effectif } \\
\text { Number }\end{array}$ & $\begin{array}{l}\text { Moyenne } \\
\text { Average }\end{array}$ & $\begin{array}{c}\text { Écart-type } \\
\text { Standard error }\end{array}$ \\
\hline 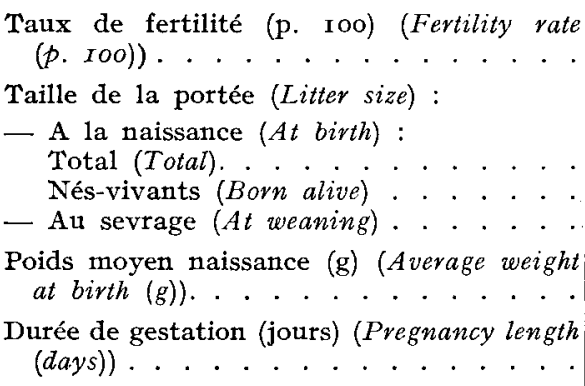 & $\begin{array}{ll}\text { I } & \text { I } 34 \\
\text { I } & \text { I } 34 \\
\text { I } & \text { I O I }\end{array}$ & $\begin{array}{r}\text { I0,28 } \\
9,47 \\
7,59 \\
\text { I } 308 \\
\\
\text { I I } 4,8\end{array}$ & $\begin{array}{r}3,02 \\
2,90 \\
2,86\end{array}$ \\
\hline
\end{tabular}

\section{b. - Influence du verrat sur la taille de la portée et le poids moyen à la naissance (fig. I)}

Les résultats de l'analyse statistique des variables sont résumés dans le tableau 2. Aucune interaction n'étant significative, l'analyse de variance a été poursuivie en faisant 1'hypothèse de la non-interaction entre les 3 facteurs "verrat ", " période de mise-bas " et " numéro de portée ".

L'effet "verrat " sur la taille de la portée est significatif à la naissance et au sevrage. Ainsi, la prolificité moyenne est de 9,47 porcelets nés vivants par portée avec une étendue de I,55 porcelet entre les verrats extrêmes. Cet écart se maintient au sevrage.

Le verrat a aussi un effet hautement significatif sur le poids moyen du porcelet à la naissance qui est, en moyenne, de $\mathrm{I} 308 \mathrm{~g}$ avec un écart de I Io $\mathrm{g}$ entre 


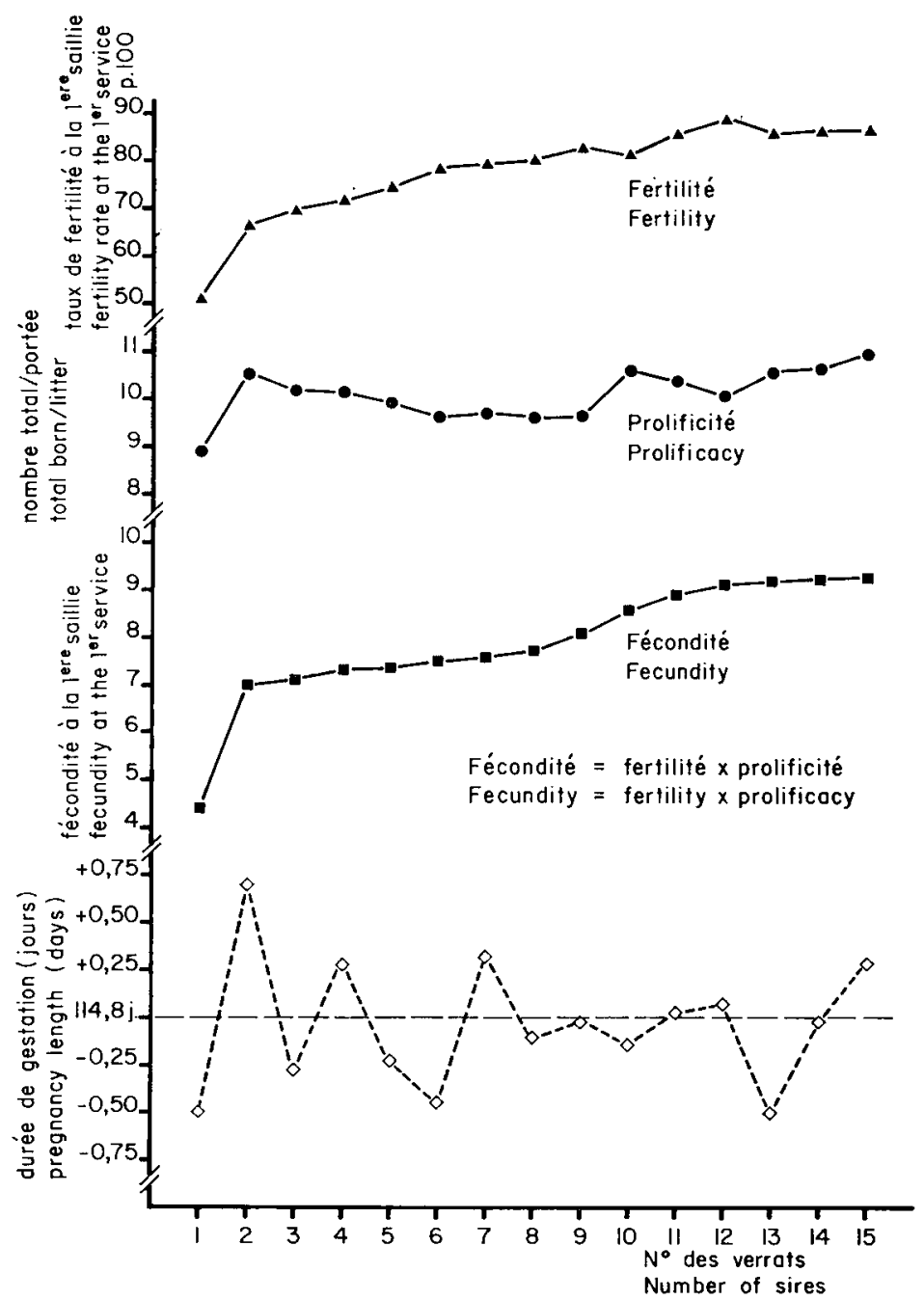

FIG. I. - Influence du "verrat" sur les paramètres de la productivité du troupeau. "Boar" effect on productivity parameters of the herd.

les verrats extrêmes. Les reproducteurs mâles les plus prolifiques engendrent des descendants moins lourds à la naissance, à l'exception d'un seul animal, pour lequel on observe à la fois une faible prolificité et des porcelets légers. Ce résultat est à rapprocher de la valeur du coefficient de corrélation hautement significatif entre le nombre de nés vivants par portée et le poids moyen du porcelet à la naissance par portée $(r=0,35)$ (tab1. 3).

I'effet du " numéro de portée " est aussi très hautement significatif pour la taille de la portée, à la naissance et au sevrage, ou pour le poids moyen des porcelets à la naissance. La période de mise-bas a, par contre, un effet seulement significatif sur le poids des porcelets à la naissance : ceux-ci sont moins lourds de novembre à avril que de mai à octobre (fig. 2). 


\section{TABIEAU 2}

Valeurs de " $F$ " dans l'analyse de la variation et signification de l'effet "verrat" " $F$ " values in the analysis of variance and significance of "boar" effect

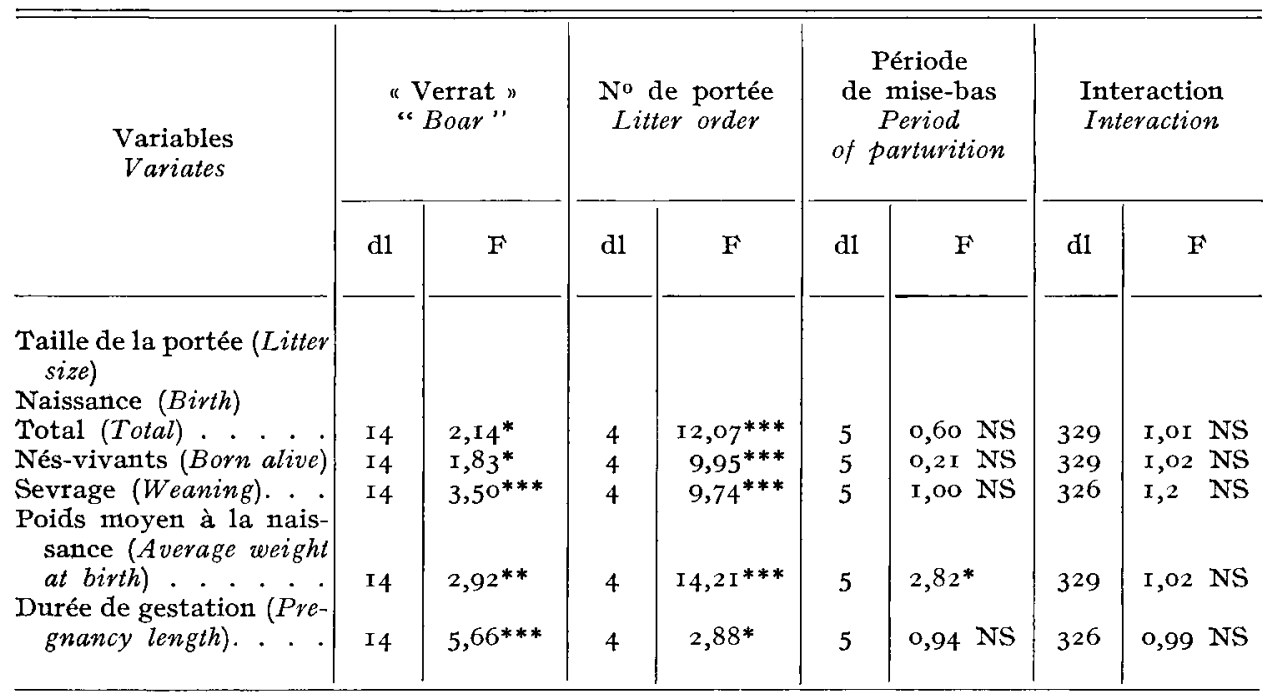

*, **, ***: F significatif aux seuils respectifs de 5 , r et o, I p. roo (F significant at the thresholds $5, I$ and 0.1 p. Ioo).

N.S. : F non significatif au seuil $5 \mathrm{p}$. Ioo (F non significant at the threshold 5 . roo).

\section{TABI,EAU 3}

Coefficients de corrélations linéaires entre les variables

Linear correlation coefficients between variates

\begin{tabular}{|c|c|c|c|c|}
\hline & $\begin{array}{l}\text { Nés vivants } \\
\text { Born alive }\end{array}$ & $\begin{array}{l}\text { Nés totaux } \\
\text { Total born }\end{array}$ & $\begin{array}{l}\text { Nombre } \\
\text { de sevrés } \\
\text { Number } \\
\text { of weaned }\end{array}$ & $\begin{array}{c}\text { Poids à la } \\
\text { naissance } \\
\text { Weight } \\
\text { at bivth }\end{array}$ \\
\hline Nés totaux $($ Total born $) .. . . .$. & $0,88 * *$ & & & \\
\hline Nombre de sevrés (Number of weaned). & $0,64^{* *}$ & $0,5^{*}$ & & \\
\hline Poids à la naissance (Weight at bivth). . & $-0,35^{* *}$ & $-0,40^{* *}$ & $-0,09^{* *}$ & \\
\hline Durée de gestation (Pregnancy length). & $-0,15^{* *}$ & $-0, \mathrm{I} 7^{* *}$ & $-0,05 \mathrm{NS}$ & $+0,09^{* *}$ \\
\hline
\end{tabular}

*,** : Significatif aux seuils respectifs 5 et I p. Ioo (Significant at the thresholds 5 and $I$ p. Ioo).

N.S. : Non significatif au seuil 5 p. roo (Non significant at the threshold 5 . 100 ). 


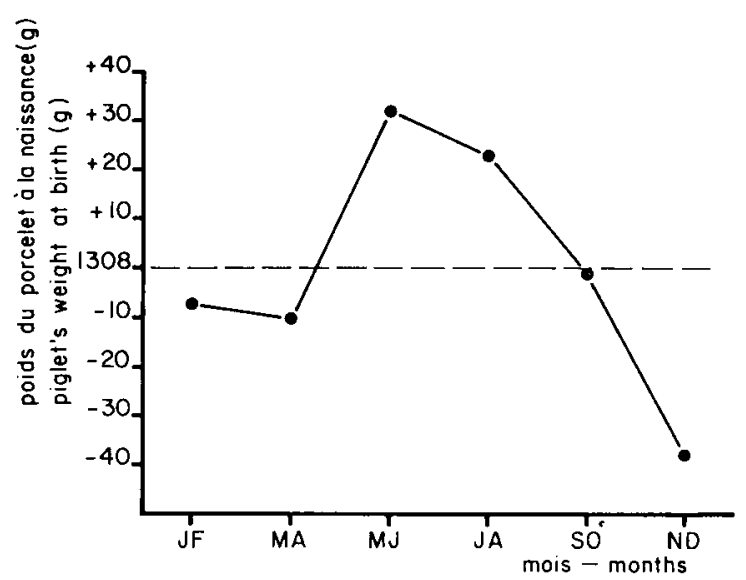

FIG. 2. - Poids moyen des porcelets à la naissance en fonction de la période de mise-bas. Average weight at birth according to the farrowing period.

\section{c. - Influence du verrat sur la durée de gestation}

La durée moyenne de la gestation est de II 4,8 jours avec un écart-type de $\mathrm{I}, 48$ jours. A partir des données corrigées pour le numéro de portée et pour la période de mise-bas, l'effet "verrat " sur la durée de gestation est très hautement significatif avec un écart de $I, 30$ jour entre les verrats extrêmes. La corrélation entre le nombre de nés vivants par portée et la durée de gestation étant hautement significative, mais faible (tab1. 3), nous avons repris l'analyse de la durée de gestation en tenant compte de la taille de la portée, afin d'estimer plus précisément l'effet " verrat ». Les résultats de cette analyse indiquent toujours un effet très hautement significatif du "verrat " avec une étendue comparable à l'étude précédente (fig. I).

L'effet " taille de portée " est aussi très hautement significatif. Le " numéro de portée " paraît après correction, sans effet sur la durée de gestation : les gestations les plus longues sont observées dans le cas des portées de plus faible taille (fig. 3).

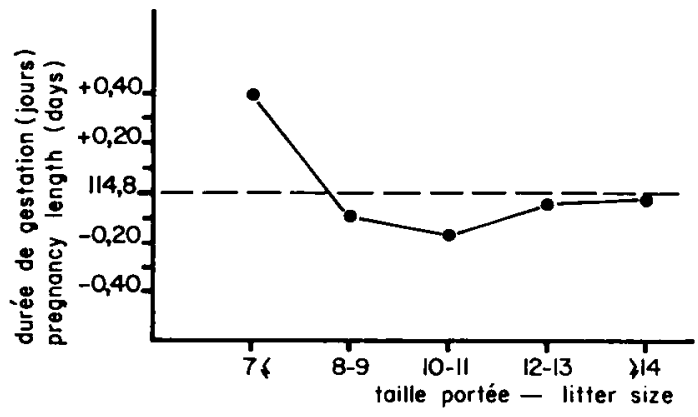

FIG. 3. - Durée de gestation en fonction de la taille de la portée. Pregnancy length according to the litter size. 


\section{Discussion}

Le verrat, utilisé en saillie naturelle, paraît avoir une importance particulière sur plusieurs paramètres de la productivité d'un troupeau. Ainsi, le mâle intervient d'abord sur le taux de fertilité, variable capitale dont dépend la durée des périodes improductives : âge à la première saillie fécondante, intervalle entre le sevrage et la nouvelle fécondation, intervalle entre le dernier sevrage et la réforme. Une subfertilité mâle peut même conduire à une réforme prématurée de certaines truies encore fécondables (Josse et al., I979). Dans notre étude, la différence de $34 \mathrm{p}$. Ioo entre les taux de fertilité des verrats extrêmes entraîne à elle seule une variabilité voisine de $I, 6$ porcelet sevré par truie et par an, estimée d'après la formule de productivité numérique des truies (LEGAULT, I978).

Le verrat intervient sur la taille de la portée à la naissance et par conséquent au sevrage. La différence estimée ici ( $I, 55$ porcelet), entre les verrats extrêmes, est un peu plus faible que celle observée par LEGAULT et OLIIVIER (I965) qui indiquent un écart de 2,5 porcelets par poriée à la naissance après insémination artificielle. L'incidence sur la productivité numérique des truies est de l'ordre de 3,5 porcelets dans le cadre de notre exemple.

Par ailleurs, en accord avec Van Oers (I964), MaIjala (I965), Du MesniL, DU Buisson et al. (I974), la fertilité et la prolificité semblent liées linéairement (coefficient de corrélation significatif, $r=+0,63$ ). Les différences de fécondité (produit des 2 paramètres) apparaissent clairement entre verrats et leurs conséquences sont d'une importance pratique considérable (fig. I). Ainsi, entre les verrats extrêmes, on observe une différence de 5,5 porcelets par truie et par an.

La variabilité de prolificité entre les verrats est constatée mais encore mal expliquée. Selon PerRy et Rowlands (rg62), si la fécondation a lieu, près de Ioo p. Ioo des ovules seraient fertilisés; le mâle pourrait donc affecter la mortalité embryonnaire. Dans l'hypothèse où cet effet se manifesterait avant l'implantation des embryons, il expliquerait aussi partiellement la liaison entre fertilité et prolificité, puisque l'état de gravidité ne peut se poursuivre que s'il y a au moins 4 embryons à s'implanter (DU MESnil DU Buisson et DAUZIER, r959). En outre, des anomalies chromosomiques sont parfois à l'origine d'une fertilité mâle réduite, comme c'est le cas en particulier pour des translocations qui aboutissent au cours de la méiose à la production de gamètes à génome incomplet (BENGT et LENNART, I964).

Le mâle semble également avoir un effet notable sur la durée de gestation de la truie, ce qui n'avait pas été mis en évidence à notre connaissance jusqu'à maintenant. Le déclenchement de la parturition est essentiellement dépendant de la maturité physiologique des fœtus (Bosc, DU MESNIL DU Buisson et LocATELI,I, I974). Le verrat pourrait done influencer les mécanismes physiologiques par les potentialités génétiques du fotus, en accord avec 1'hypothèse formulée par JAFAR, Chapman et CASIDA (I950) chez les bovins.

Enfin, d'autres effets ont été constatés dans cette étude : les variations saisonnières sont peu marquées sur la prolificité qui apparaît un peu plus élevée pour les portées d'hiver. Des différences plus importantes avaient été notées dans l'enquête d'OLLIVIER et LEGAULT (r967).

Enfin, la durée de gestation, relativement constante dans l'espèce porcine, peut aussi varier en raison inverse de la taille de la portée, et ceci en accord avec 
les résultats de LECyk et al. (I976) et Aumaitre, Deglaire et LEBOST (I976). I a relation entre la prolificité et la durée de gestation est connue dans d'autres espèces, en particulier chez les ovins (Bosc et Cornu, I976).

\section{Conclusion}

Cette étude souligne l'importance de la variabilité du pouvoir fécondant entre les verrats utilisés en saillie naturelle. Ainsi, nous avons observé une différence de $34 \mathrm{p}$. Ioo pour le taux de fertilité et de $\mathrm{I}, 55$ porcelet en moyenne par portée entre les verrats extrêmes. L'effet du mâle sur la productivité numérique d'un troupeau étant démontré, il reste à identifier les paramètres susceptibles de modifier l'aptitude du reproducteur à une carrière performante.

Accepté pour publication en avril 1979.

\section{Remerciements}

L'auteur tient à remercier MM. A. AUMAITre et C. LEGAULir pour l'aide et les suggestions apportées lors de l'interprétation des résultats et de la tédaction de ce texte.

\section{Summary}

Infuence of the boar on the main productivity parameters of the herd and on the pregnancy length

The effect of the boar on the main productivity parameters of a Large White herd was estimated on I,74I matings and I,I34 litters. These data were obtained from boars used in natural mating in the experimental herd of " La Minière" during the period I972-I978.

The boar effect was highly significant on fertility and significant on litter size at birth and at weaning (fig. I). The differences concerning fertility led to a variability of I.6 piglets weaned per sow and per year, those concerning prolificacy resulted in a variability of 3.5 piglets and the combination of fertility and prolificacy differences between boars was $5 \cdot 5$ piglets.

The boar effect was also highly significant on the pregnancy length of the sows with a variability of I.3o day.

\section{Références bibliographiques}

Arbonnier P., I966. L'analyse de l'information. Aperçu théorique et application à la loi multinomiale. Ann. Sci. For. XXIII, 4, 949-IOI 7 .

Aumaitra A., Deglaire B., Lebost J., I979. Primaturité de la mise bas chez la truie et signification du poids à la naissance du porcelet. Ann. Biol. anim. Bioch. Biophys., 19 (IB), $267^{-275}$.

BARBOSA A. S., I962. Some factors affecting litter size and weight and pigling variability at 90 days in Berkshire and Duroc-Jersey pigs bred at the "Diaulas Abreu " college of Agricultural Technology. Arq. Esc. Veter. Univ. Minas. Gerais, 13, 255-29I (in Anim. Breed. Abstr., 32, 1204 ).

BENGT H., LENNART B., I964. Translocation heterozygosity in a boar. Heveditas, 52, I66-I 70. 
BOSC M. J., DU MESNI, DU BuISson F., Locater, I A., I974. Mise en évidence d'un contrôle foetal de la parturition chez la truie. Interaction avec la fonction lutéale. C. R. Acad. Sci. Paris, sér. $D, \mathbf{2 7 8}$, I 507-15 I0.

Bose M. J., Cornu C., I976. Étude des facteurs affectant les conditions de mise-bas et la survie des agneanx. Journées Rech. Ov. Capr. I.N.R.A.-I.T.O.V.I.C., Paris, 2, 306-321.

Dzaparidze, I935. Studies on the estimation of fertility and weight at birth and at four weeks in the Hannover - Braun - schweig land pig. Züchtungskunde, 10, 208-2I 7 (in Anim. Breed. Abstr., 4, 203).

Eurostat, 1978. Statistiques mensuelles de la viande. C.E.E., éd. Bruxelles (6).

JAFAR S., Chapman A. B., CASIDA I, E., I95o. Causes of variation in length of gestation in daity cattle. J. Anim. Sci., 9, 593-60т.

Josse J., Le Denmat M., Martinat-Botte F., Saulnier J., Vannier P., Vaudelet J. C., 1979. A propos d'une enquête sur les causes de réformes des truies. Journées Rech. Porcine en France, 11, 355-364, Paris, I.N.R.A.-I.T.P. éd.

KRALIINGER H. F., SchorT A., I934. The influence of the boar on litter size. Anim. Breed. Abstr., 2, I27.

LECYK K., NozdRyN-Platnicki J., WALkIEwiez A., ZIN M., I976. Effect of duration of pregnancy in Large White sows on litter size and vitality. Roczn. Nauk. Rolnic., Ser. B, 97, IO3-III.

IEGAULT C., I970. Étude statistique et génétique des performances d'élevage des truies de race Large White. Ann. Génét. Sél. anim., 2 (2), 209-227.

LEgauli C., 1978. Génétique et reproduction chez le porc. Journées Rech. Porcine en France, 10, 43-6o, Paris, I.N.R.A.-I.T.P. éd.

LEGAULT C., OLIIVIER L., I965. Résultats préliminaires concernant l'influence du verrat sur la taille de la portée en insémination artificielle. Ann. Zootech., 14, 40I-408.

MaIJALA K., I965. Communication aux Journées d'Études de la F.E.Z., Noordwijk. Commission de Génétique.

Du Mesnil du Buisson F., DAuzifR L., 1959. Contrôle mutuel de l'utérus et de l'ovaire chez la truie. Ann. Zootech., 8, série D, supp. I47-r 59 .

Du Mesnim, du Buisson F., Mulanvove B,, Bariteau F., Legaula C., r974. Facteuts de variation de la production et de la qualité de la semence du verrat : effets saisontniers, héritabilité, corrélations entre variables. Journées Rech. Porcine en France, 6, 63-7o, Paris, I.N.R.A.I.T.P. éd.

Musson A. L., I946. The influence of the boar on litter size. J. Anim. Sci., 5, 4r8 (Abstr.).

OLLIVIER L., IEGAULT C., I967. L'influence directe du verrat sur la taille et le poids des portées obtenues par insémination artificielle. Ann. Zootech., 16, 247-254.

Perry J. S., Rowlands I. W., 1962. Early pregnancy in the pig. J. Reprod. Fertil., 4, I75-I88.

REDDY V. B., LASIEY J. F., MAYER D. T., I958. Genetic aspects of reproduction in swine. University of Missouri Res. Bull., 666.

Rico C., MENchaca M., I975. Reproductive performance of the Duroc breed in Cuba. I. Effect of different sources of variation. Cuban Journal of Agricultural Science, 9, I35-I48.

SMIrH W. C., TOF' $\mathrm{C}$. R., r978. A note on the comparative performance of pigs sired by Pietrain $\times$ Large White boars. Anim. Prod., 27, I2 I-I 24.

SkJERvold H., I963. To what extent do boar affect the litter size. Meld. Norges Landbrukshogsk., 42, I I pages.

STEVENS W. L., I95 I. Asymptotic regression. Biometvics, 7, 247-267.

VAN OERS J. P. S., 1964. De invloed van de beer op de Worpgrootte. Veeteelt en Zuivelberichten, 6, 5 I I -520 .

Wilson S. P., Whateley J. A. Jr, Whiteamon J. V., Morrison R. D., ig62. Influence of sire and line of breeding on sow productivity. J. Anim. Sci., 21, I I9-122. 